\title{
On The Exact Solutions to Conformable Time Fractional Equations in EW Family Using Sine-Gordon Equation Approach
}

\author{
Alper Korkmaz ${ }^{a, *}$ Ozlem Ersoy Hepson ${ }^{b}$, Kamyar Hosseini $^{c}$, \\ Hadi Rezazadeh ${ }^{d}$, Mostafa Eslami ${ }^{e}$ \\ ${ }^{a}$ Çankırı Karatekin University, Department of Mathematics, 18200, Çankırı, Turkey. \\ ${ }^{b}$ Eskişehir Osmangazi University, Department of Computer and Mathematics, Eskişehir, Turkey. \\ ${ }^{c}$ Department of Mathematics, Rasht Branch, Islamic Azad University, Rasht, Iran. \\ ${ }^{d}$ Faculty of Engineering Technology, Amol University of Special Modern Technologies, Amol, Iran. \\ ${ }^{e}$ Department of Mathematics, Faculty of Mathematical Sciences, University of Mazandaran, Babolsar, Iran.
}

\begin{abstract}
New exact solutions to conformable time fractional EW and modified EW equations are constructed by using Sine-Gordon expansion approach. The fractional traveling wave transform and homogeneous balance have significant roles in the solution procedure. The predicted solution is of the form of some finite series of multiplication of powers of cos and sin functions. The relation among trigonometric and hyperbolic functions in sense of Sine-Gordon expansion gives opportunity to construct the solutions in terms of hyperbolic functions.
\end{abstract}

Keywords: Sine-Gordon Expansion Method; Conformable time fractional EW Equation; Conformable time fractional modified EW Equation; Exact Solution, Traveling Solution.

MSC2010: 35C07;35R11;35Q53.

PACS: 02.30.Jr; 02.70.Wz; 04.20.Jb

*alperkorkmaz7@gmail.com 


\section{Introduction}

In the recent several decades, many developments in computer algebra field have been witnessed. These developments have also lead to solve many nonlinear PDE problems symbolically due to faster symbolic algebraic manipulations when compared with study with pencil. Moreover, more methods have been introduced and implemented to lots of nonlinear PDEs. The prominent ones of those methods are various types of simple ansatz techniques with positive integer powers [1-3]. First integral method [4-6] is an alternative technique to simple ansatzes to construct exact solutions to nonlinear PDEs. Exp function method $[7,8]$ assumes the predicted solutions as a finite series of some particular functions. $\left(G^{\prime} / G\right)$ expansion method $[9,10]$ is an alternative that approaches the solution with a finite power series of a function satisfying a particular ODE. Trigonometric and hyperbolic type solutions to nonlinear PDEs can be determined by implementation of sine-cosine approach [11-13].

Later on, the implementations of those methods have been extended to solutions of fractional nonlinear PDEs [14-23]. Existence of some particular traveling wave transforms has given opportunity to exact solutions to fractional PDEs with nonlinear terms. Physical interpretation of fractional derivatives has been comprehended deeply by examining plots of solutions in both time fractional and spacetime fractional cases.

Even though there exist various definitions of fractional derivative in the literature, we focus time fractional equal width equation (fEWE)

$$
D_{t}^{\gamma} u+p u u_{x}+q D_{t}^{\gamma} u_{x x}=0, t>0
$$

and time fractional modified equal width equation (fmEWE)

$$
D_{t}^{\gamma} u+p u^{2} u_{x}+q D_{t}^{\gamma} u_{x x}=0, t>0
$$

where $u$ is function of the independent variables $t$ and $x, p$ and $q$ are real parameters. One should note that $D_{t}^{\gamma}$ is conformal fractional derivative operator defined in [24]. The original of the fEWE with integer order appeared in the study of Morrison et al. [25]. This equation (when $\gamma=1$ ) has a simple relation with the well-known RLW or BBM equation. The integer ordered EWE has singular wave solutions expressed in forms of powers of sech function [25].

\section{Fractional Derivative in Conformable Sense}

$\gamma$. th order conformable derivative of a function $y=y(t)$ is defined as

$$
D_{t}^{\gamma}(y(t))=\lim _{\tau \rightarrow 0} \frac{y\left(t+\tau t^{1-\gamma}\right)-y(t)}{\tau}, t>0, \gamma \in(0,1]
$$


where $y=y(t):[0, \infty) \rightarrow \mathbb{R}[24]$. This recent definition of fractional derivative satisfies the following properties.

Theorem 1 Let $u=u(t)$ and $y=y(t)$ are $\gamma$-differentiable for all positive $t$. Then,

- $D_{t}^{\gamma}\left(d_{1} u(t)+d_{2} y(t)\right)=d_{1} D_{t}^{\gamma}(u(t))+d_{2} D_{t}^{\gamma}(y(t))$

- $D_{t}^{\gamma}\left(t^{m}\right)=m t^{m-\gamma}, \forall m \in \mathbb{R}$

- $D_{t}^{\gamma}\left(d_{3}\right)=0$, for all constant function $u(t)=d_{3}$

- $D_{t}^{\gamma}(u(t) y(t))=u(t) D_{t}^{\gamma}(y(t))+y(t) D_{t}^{\gamma}(u(t))$

- $D_{t}^{\gamma}\left(\frac{u(t)}{y(t)}\right)=\frac{y(t) D_{t}^{\gamma}(u(t))-u(t) D_{t}^{\gamma}(y(t))}{y^{2}(t)}$

- $D_{t}^{\gamma}(u(t))=t^{1-\gamma} \frac{d u(t)}{d t}$

for $\forall d_{1}, d_{2}, d_{3} \in \mathbb{R}[26,27]$.

Many significant properties like the chain rule, Laplace transform and Taylor series expansion are valid for this definition of fractional derivative operator [28].

Theorem 2 Let $u=u(t)$ be an $\gamma$-conformable differentiable function. Then,

$$
D_{t}^{\gamma}(u \circ y)(t)=t^{1-\gamma} y^{\prime}(t) u^{\prime}(y(t))
$$

where $y=y(t)$ is defined in the range of $u(t)$ and differentiable in the classical sense.

\section{Sine-Gordon Equation (SGE) Approach}

Consider the one dimensional SGE of the form

$$
\frac{\partial^{2} u}{\partial x^{2}}-\frac{\partial^{2} u}{\partial t^{2}}=m^{2} \sin u, \quad m \text { is constant }
$$

where $u=u(x, t)$. The traveling wave transform in one dimension $u(x, t)=U(\eta)$ with $\eta=a\left(x-\nu t^{\alpha} / \alpha\right)$ reduces the SGE to the ODE

$$
\frac{d^{2} U}{d \eta^{2}}=\frac{m^{2}}{a^{2}\left(1-\nu^{2}\right)} \sin U
$$


where $\nu$ indicates the velocity of the wave described in the traveling wave transform [29]. Some algebra and substitutions converts the transformed equation to

$$
\left(\frac{d(U / 2)}{d \eta}\right)^{2}=\frac{m^{2}}{a^{2}\left(1-\nu^{2}\right)} \sin ^{2} U / 2+C
$$

where $C$ is integration constant and assumed zero. Assume that $\tilde{H}(\eta)=U(\eta) / 2$ and $m^{2} /\left(a^{2}\left(1-\nu^{2}\right)\right)=1$. Then, (7) takes the form

$$
\frac{d(\tilde{H})}{d \eta}=\sin \tilde{H}
$$

Thus, (8) gives the following relations

$$
\sin \tilde{H}(\eta)=\left.\frac{2 c e^{\eta}}{c^{2} e^{2 \eta}+1}\right|_{c=1}=\operatorname{sech} \eta
$$

or

$$
\cos \tilde{H}(\eta)=\left.\frac{c^{2} e^{2 \eta}-1}{c^{2} e^{2 \eta}+1}\right|_{c=1}=\tanh \eta
$$

where $c \neq 0$ is integral constant.

On the other hand, the governing fractional PDE

$$
\Omega\left(u, D_{t}^{\gamma} u, u_{x}, D_{t t}^{2 \gamma} u, u_{x x}, \ldots\right)=0
$$

is reduced to an ODE of the form

$$
\tilde{\Omega}\left(U, U^{\prime}, U^{\prime \prime}, \ldots\right)=0
$$

by using the fractional traveling wave transform $u(x, t)=U(\eta)$ with the transform variable $\eta=a\left(x-\nu t^{\gamma} / \gamma\right)$. Then, a predicted solution to (12) of the form

$$
U(\eta)=A_{0}+\sum_{i=1}^{s} \tanh ^{i-1}(\eta)\left(B_{i} \operatorname{sech} \eta+A_{i} \tanh \eta\right)
$$

is constructed. This solution can be expressed in terms of $w$ as

$$
U(\tilde{H})=A_{0}+\sum_{i=1}^{s} \cos ^{i-1}(\tilde{H})\left(B_{i} \sin \tilde{H}+A_{i} \cos \tilde{H}\right)
$$

due to the relations $(9)-(10)$. The solution process begins by finding $s$ by the help of homogenous balance procedure for (12). The determination of $s$ gives the exact power of the solution in series of trigonometric and hyperbolic functions. 
After substitution of the predicted solution (14) into (12) and some algebra, the coefficients of powers of $\sin \tilde{H} \cos \tilde{H}$ are assumed as zero. Thus, an algebraic system of equations are obtained. This system is solved for the predicted solution coefficients $A_{0}, A_{1}, B_{1}, \ldots$ and one or both of transform coefficients $a, \nu$. If one can find a solution satisfying the conditions given above, then, the solutions are constructed by using (9) - (10) and $\eta$. The final forms of the solutions are written by substitution of $x$ and $t$ with the relations of transform parameters $a$ and $\nu$ instead of $\eta$.

\section{Solutions to the conformable time fractional EWE}

The time fractional EWE of the form (1) is reduced to the ODE

$$
-a \nu U+\frac{1}{2} p a U^{2}-q \nu a^{3} U^{\prime \prime}=C
$$

where $C$ is constant by the use of fractional traveling wave transform. The balance between $U^{2}$ and $U^{\prime \prime}$ gives $s=2$. Thus, the predicted solution is constructed as

$$
U(\eta)=A_{0}+A_{1} \cos \tilde{H}+B_{1} \sin \tilde{H}+A_{2} \cos ^{2} \tilde{H}+B_{2} \cos \tilde{H} \sin \tilde{H}
$$

Substitution of the predicted solution (16) into (15) gives

$$
\begin{aligned}
& -(\cos (\tilde{H}(\eta)))^{2} a \nu A_{2}-\cos (\tilde{H}(\eta)) a \nu A_{1}-\sin (\tilde{H}(\eta)) a \nu B_{1}+1 / 2(\cos (\tilde{H}(\eta)))^{4} a p A_{2}{ }^{2}+1 / 2(\cos (\tilde{H}(\eta)))^{2} a p A_{1}{ }^{2} \\
& +1 / 2(\sin (\tilde{H}(\eta)))^{2} a p B_{1}{ }^{2}-C+2 \cos (\tilde{H}(\eta))(\sin (\tilde{H}(\eta)))^{2} a^{3} \nu q A_{1}-(\cos (\tilde{H}(\eta)))^{3} a^{3} \nu q B_{2} \sin (w(\eta)) \\
& -(\cos (\tilde{H}(\eta)))^{2} a^{3} \nu q B_{1} \sin (\tilde{H}(\eta))+4(\cos (\tilde{H}(\eta)))^{2}(\sin (\tilde{H}(\eta)))^{2} a^{3} \nu q A_{2}+5 \cos (\tilde{H}(\eta))(\sin (\tilde{H}(\eta)))^{3} a^{3} \nu q B_{2} \\
& -2(\sin (\tilde{H}(\eta)))^{4} a^{3} \nu q A_{2}+(\sin (w(\eta)))^{3} a^{3} \nu q B_{1}+1 / 2 a p A_{0}{ }^{2}-a \nu A_{0}-\cos (\tilde{H}(\eta)) \sin (\tilde{H}(\eta)) a \nu B_{2} \\
& +1 / 2(\cos (\tilde{H}(\eta)))^{2}(\sin (\tilde{H}(\eta)))^{2} a p B_{2}{ }^{2}+(\cos (\tilde{H}(\eta)))^{3} a p A_{1} A_{2}+(\cos (\tilde{H}(\eta)))^{2} a p A_{0} A_{2}+\cos (\tilde{H}(\eta)) a p A_{0} A_{1} \\
& +\sin (\tilde{H}(\eta)) a p A_{0} B_{1}+(\cos (\tilde{H}(\eta)))^{3} \sin (\tilde{H}(\eta)) a p A_{2} B_{2}+(\cos (\tilde{H}(\eta)))^{2} \sin (\tilde{H}(\eta)) a p A_{1} B_{2} \\
& +(\cos (\tilde{H}(\eta)))^{2} \sin (w(\eta)) a p A_{2} B_{1}+\cos (\tilde{H}(\eta))(\sin (\tilde{H}(\eta)))^{2} a p B_{1} B_{2} \\
& +\cos (\tilde{H}(\eta)) \sin (\tilde{H}(\eta)) a p A_{0} B_{2}+\cos (\tilde{H}(\eta)) \sin (\tilde{H}(\eta)) a p A_{1} B_{1}=0
\end{aligned}
$$

Following usage of some trigonometric identities and simplifications, the coefficients of multiplications of powers of sin and cos functions are equated to zero. The solution of this resultant algebraic system of equations for $A_{i}, i=1,2,3, B_{j}, j=$ 
1,2 and the transform coefficients $a$ and/or $\nu$ gives

$$
\begin{aligned}
& \nu=\sqrt{\frac{2 C p}{q^{2} a^{5}-a}}, \quad A_{0}=-\frac{\left(5 a^{2} q-1\right) \sqrt{\frac{2 C p}{q^{2} a^{5}-a}}}{p}, \quad A_{1}=0, \quad A_{2}=\frac{6 a^{2} q \sqrt{\frac{2 C p}{q^{2} a^{5}-a}}}{p}, \quad B_{1}=0, \quad B_{2}=6 q a \sqrt{-\frac{2 a C}{p q^{2} a^{4}-p}} \\
& \nu=\sqrt{\frac{2 C p}{q^{2} a^{5}-a}}, \quad A_{0}=-\frac{\left(5 a^{2} q-1\right) \sqrt{\frac{2 C p}{q^{2} a^{5}-a}}}{p}, \quad A_{1}=0, \quad A_{2}=\frac{6 a^{2} q \sqrt{\frac{2 C p}{q^{2} a^{5}-a}}}{p}, \quad B_{1}=0, \quad B_{2}=-6 q a \sqrt{-\frac{2 a C}{p q^{2} a^{4}-p}} \\
& \nu=-\sqrt{\frac{2 C p}{q^{2} a^{5}-a}}, \quad A_{0}=-\frac{\left(5 a^{2} q-1\right) \sqrt{\frac{2 C p}{q^{2} a^{5}-a}}}{p}, \quad A_{1}=0, \quad A_{2}=\frac{6 a^{2} q \sqrt{\frac{2 C p}{q^{2} a^{5}-a}}}{p}, \quad B_{1}=0, \quad B_{2}=6 q a \sqrt{-\frac{2 a C}{p q^{2} a^{4}-p}} \\
& \nu=-\sqrt{\frac{2 C p}{q^{2} a^{5}-a}}, \quad A_{0}=-\frac{\left(5 a^{2} q-1\right) \sqrt{\frac{2 C p}{q^{2} a^{5}-a}}}{p}, \quad A_{1}=0, \quad A_{2}=\frac{6 a^{2} q \sqrt{\frac{2 C p}{q^{2} a^{5}-a}}}{p}, \quad B_{1}=0, \quad B_{2}=-6 q a \sqrt{-\frac{2 a C}{p q^{2} a^{4}-p}} \\
& \nu=\sqrt{\frac{2 C p}{16 q^{2} a^{5}-a}}, \quad A_{0}=-\frac{\left(8 a^{2} q-1\right) \sqrt{\frac{2 C p}{16 q^{2} a^{5}-a}}}{p}, \quad A_{1}=0, \quad A_{2}=\frac{12 a^{2} q \sqrt{\frac{2 C p}{16 q^{2} a^{5}-a}}}{p}, \quad B_{1}=0, \quad B_{2}=0 \\
& \nu=-\sqrt{\frac{2 C p}{16 q^{2} a^{5}-a}}, \quad A_{0}=\frac{\left(8 a^{2} q-1\right) \sqrt{\frac{2 C p}{16 q^{2} a^{5}-a}}}{p}, \quad A_{1}=0, \quad A_{2}=-\frac{12 a^{2} q \sqrt{\frac{2 C p}{16 q^{2} a^{5}-a}}}{p}, \quad B_{1}=0, \quad B_{2}=0
\end{aligned}
$$

Using these values of coefficients, the solutions to fractional EWE (1) are expressed explicitly as

$u_{1,2,3,4}(x, t)=-\frac{\left(5 a^{2} q-1\right) \sqrt{\frac{2 C p}{q^{2} a^{5}-a}}}{p}+\frac{6 a^{2} q \sqrt{\frac{2 C p}{q^{2} a^{5}-a}}}{p} \tanh ^{2} a\left(x-\nu \frac{t^{\gamma}}{\gamma}\right) \pm 6 q a \sqrt{-\frac{2 a C}{p q^{2} a^{4}-p}} \tanh a\left(x-\nu \frac{t^{\gamma}}{\gamma}\right) \operatorname{sech} a\left(x-\nu \frac{t^{\gamma}}{\gamma}\right)$

for $\nu= \pm \sqrt{(2 C p) /\left(q^{2} a^{5}-a\right)}$. Similarly,

$$
u_{5,6}(x, t)=\mp \frac{\left(8 a^{2} q-1\right) \sqrt{\frac{2 C p}{16 q^{2} a^{5}-a}}}{p} \pm \frac{12 a^{2} q \sqrt{\frac{2 C p}{16 q^{2} a^{5}-a}}}{p} \tanh ^{2} a\left(x-\nu \frac{t^{\gamma}}{\gamma}\right)
$$

for $\nu= \pm \sqrt{(2 C p) /\left(16 q^{2} a^{5}-a\right)}$. We demonstrate the solution $u_{5}(x, t)$ for some particular choices of parameters as $a=-1 / 2, q=1 / 2, p=-1, C=-1$. These parameters gives the particular form of the solution as

$$
u(x, t)=\sqrt{12} \tanh ^{2}\left(-\frac{x}{2}-\frac{\sqrt{48} t^{\gamma}}{6 \gamma}\right)
$$

This solution is simulated for various values of $\gamma$ in Fig 1(a)-1(d). This solution models propagation of a negative pulse along the negative $x$-direction without changing its shape and height. When $\gamma=0.20$, the initial pulse of propagates faster at the beginning but after some time it slows down, Fig 1(a). Increase of $\gamma$ towards 1 directly effects the velocity of the pulse and the propagation velocity approaches some constant value. Finally, $\gamma=1$ causes a constant velocity, Fig $1(\mathrm{~d})$. The shape and height of the initial pulse are conserved during propagation. 


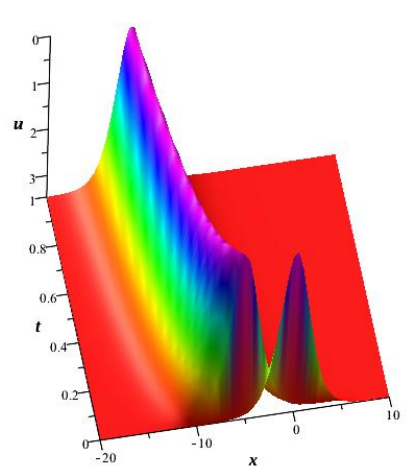

(a) $\gamma=0.20$

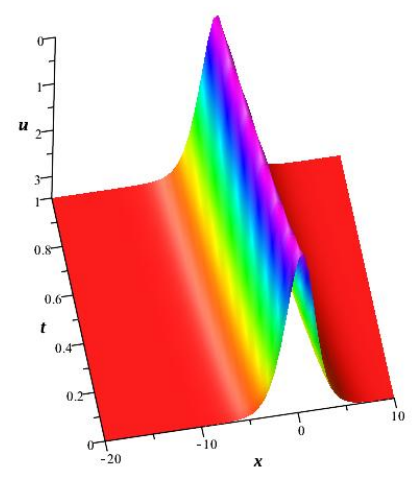

(c) $\gamma=0.70$

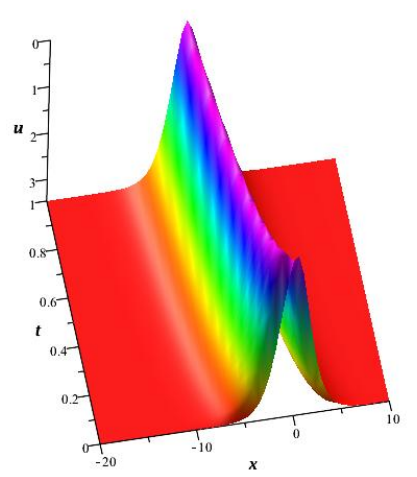

(b) $\gamma=0.40$

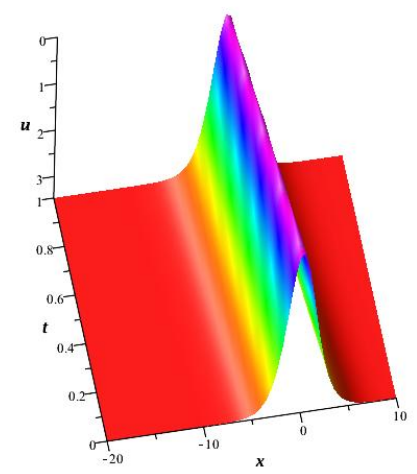

(d) $\gamma=1$

Figure 1: The solution $u_{5}(x, t)$ for various derivative orders

\section{Solutions to the conformable time fractional mEWE}

The fractional traveling wave transform reduces the mEWE to

$$
-a \nu U+\frac{1}{3} p a U^{3}-q \nu a^{3} U^{\prime \prime}=C
$$

where $C$ is integration constant. The homogeneous balance between $U^{3}$ and $U^{\prime \prime}$ gives $s=1$. Thus, the predicted solution takes the form

$$
U(\tilde{H})=A_{0}+A_{1} \cos \tilde{H}+B_{1} \sin \tilde{H}
$$


in terms of $w$. Substitution of this solution into (22)gives

$$
\begin{aligned}
& 2 \cos (\tilde{H}(\eta))(\sin (\tilde{H}(\eta)))^{2} a^{3} \nu q A_{1}+(\sin (\tilde{H}(\eta)))^{3} a^{3} \nu q B_{1}+1 / 3(\cos (\tilde{H}(\eta)))^{3} a p A_{1}{ }^{3} \\
& +(\cos (\tilde{H}(\eta)))^{2} \sin (\tilde{H}(\eta)) a p{A_{1}}^{2} B_{1}+\cos (\tilde{H}(\eta))(\sin (\tilde{H}(\eta)))^{2} a p A_{1} B_{1}{ }^{2} \\
& +1 / 3(\sin (\tilde{H}(\eta)))^{3} a p B_{1}^{3}+(\cos (\tilde{H}(\eta)))^{2} a p A_{0} A_{1}^{2}+2 \cos (\tilde{H}(\eta)) \sin (\tilde{H}(\eta)) a p A_{0} A_{1} B_{1} \\
& +(\sin (\tilde{H}(\eta)))^{2} a p A_{0} B_{1}{ }^{2}+\cos (\tilde{H}(\eta)) a p A_{0}{ }^{2} A_{1}+\sin (\tilde{H}(\eta)) a p A_{0}^{2} B_{1}+1 / 3 a p A_{0}{ }^{3} \\
& -(\cos (\tilde{H}(\eta)))^{2} a^{3} \nu q B_{1} \sin (\tilde{H}(\eta))-\cos (\tilde{H}(\eta)) a \nu A_{1}-\sin (\tilde{H}(\eta)) a \nu B_{1}-a \nu A_{0}-C=0
\end{aligned}
$$

Using some trigonometric identities and simplifications, we equate the coefficients of powers of multiplications of cos and sin functions and the constants to zero. Thus, we find a system of algebraic equations. Solution of this system for $a, \nu$, $A_{0}, A_{1}, B_{1}$ and $C$ gives

$$
\begin{aligned}
& a=\sqrt{-\frac{1}{q}}, A_{0}=0, A_{1}=0, B_{1}=\sqrt{\frac{6 \nu}{p}} \\
& a=\sqrt{-\frac{1}{q}}, A_{0}=0, A_{1}=0, B_{1}=-\sqrt{\frac{6 \nu}{p}} \\
& a=-\sqrt{-\frac{1}{q}}, A_{0}=0, A_{1}=0, B_{1}=\sqrt{\frac{6 \nu}{p}} \\
& a=-\sqrt{-\frac{1}{q}}, A_{0}=0, A_{1}=0, B_{1}=-\sqrt{\frac{6 \nu}{p}}
\end{aligned}
$$

for $C=0$ and arbitrary $\nu$. Thus, the solutions to (2) are constructed as

$$
u_{7,8}= \pm \sqrt{\frac{6 \nu}{p}} \operatorname{sech}\left(\sqrt{-\frac{1}{q}}\left(x-\nu \frac{t^{\gamma}}{\gamma}\right)\right)
$$

and

$$
u_{9,10}= \pm \sqrt{\frac{6 \nu}{p}} \operatorname{sech}\left(-\sqrt{-\frac{1}{q}}\left(x-\nu \frac{t^{\gamma}}{\gamma}\right)\right)
$$

A particular form of the solution $u_{9}(x, t)$ is determined as

$$
u(x, t)=\sqrt{30} \operatorname{sech}\left(x-\frac{5 t^{\gamma}}{\gamma}\right)
$$

by using the parameters as $\nu=5, q=-1, p=1$. This solution models propagation of an initial positive pulse along $x$-axis. As time proceeds, the initial pulse travels 
by keeping its shape and height. This particular solution is depicted for various values of $\gamma$ in Fig 2(a)-2(d) in some finite domain of $x$ and $t$. When $\gamma$ is 0.20 and 0.40 , we observe that the pulse moves faster at the beginning, but slows down as time goes, Fig 2(a)-2(b). The change of velocity is hardly observed for $\gamma=0.70$, Fig 2(c). The velocity is constant with the choice $\gamma=1$, Fig $2(\mathrm{~d})$.

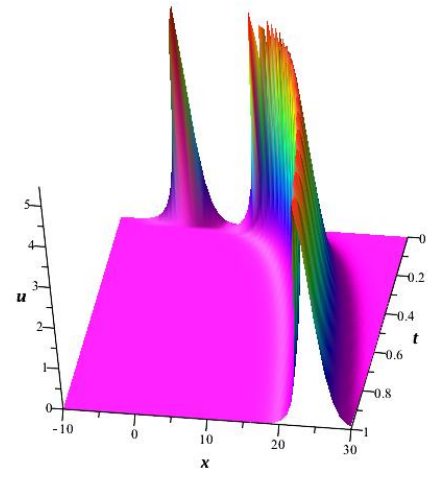

(a) $\gamma=0.20$

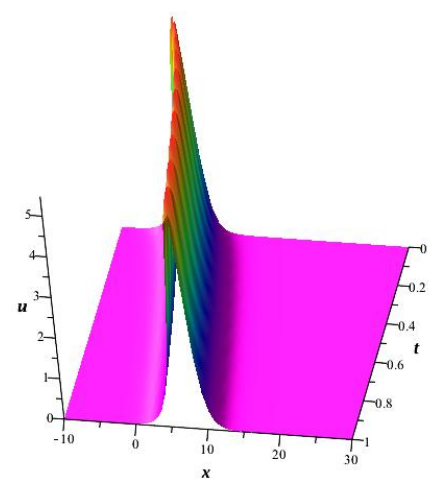

(c) $\gamma=0.70$

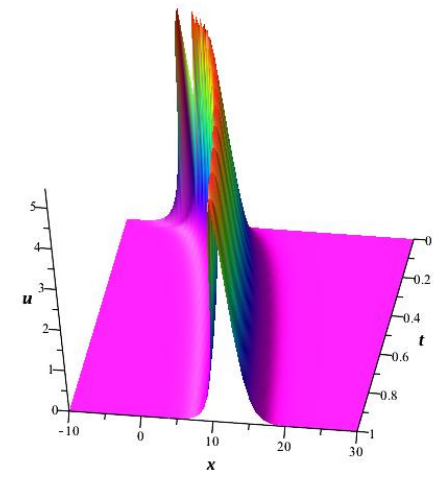

(b) $\gamma=0.40$

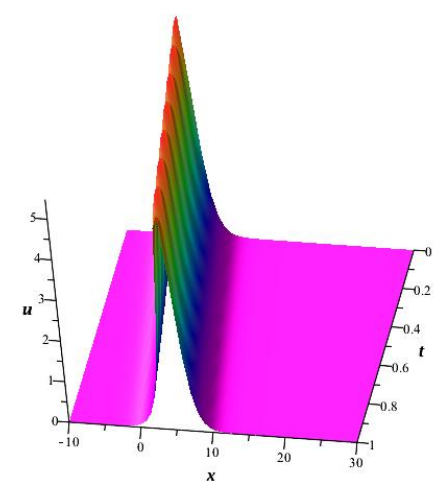

(d) $\gamma=1$

Figure 2: The solution $u_{9}(x, t)$ for various derivative orders

\section{Conclusion}

SGE method was implemented to extract the exact solutions to conformable fEWE and fmEWE equations. Fractional traveling wave transform reduces the governing equation to some ODEs. The homogeneous balance principle has a significant role to determine the degree of the series of the solutions. The solutions consist of multiplications of powers of sech and tanh functions. Once the degree and 
structure of predicted solution was determined, it was directly substituted into the resultant ODE. The algebraic system constructed by equating the coefficients to zero was solved to determine the coefficients of the predicted solution and the parameters of fractional traveling wave transform. Illustrations of some particular forms of the solutions indicate the effects of $\gamma$ to the initial pulses. 


\section{References}

[1] Guner, O., Bekir, A., \& Karaca, F. (2016). Optical soliton solutions of nonlinear evolution equations using ansatz method. Optik-International Journal for Light and Electron Optics, 127(1), 131-134.

[2] Bekir, A., Cevikel, A. C., Guner, O., \& San, S. (2014). Bright and dark soliton solutions of the $(2+1)$-dimensional evolution equations. Mathematical Modelling and Analysis, 19(1), 118-126.

[3] Bekir, A., \& Guner, O. (2013). Topological (dark) soliton solutions for the Camassa-Holm type equations. Ocean Engineering, 74, 276-279.

[4] Taghizadeh, N., Mirzazadeh, M., \& Farahrooz, F. (2011). Exact soliton solutions of the modified KdV-KP equation and the Burgers-KP equation by using the first integral method. Applied Mathematical Modelling, 35(8), 3991-3997.

[5] Tascan, F., Bekir, A., \& Koparan, M. (2009). Travelling wave solutions of nonlinear evolution equations by using the first integral method. Communications in Nonlinear Science and Numerical Simulation, 14(5), 1810-1815.

[6] Abbasbandy, S., \& Shirzadi, A. (2010). The first integral method for modified Benjamin-Bona-Mahony equation. Communications in Nonlinear Science and Numerical Simulation, 15(7), 1759-1764.

[7] Bekir, A., \& Boz, A. (2008). Exact solutions for nonlinear evolution equations using Exp-function method. Physics Letters A, 372(10), 1619-1625.

[8] Bekir, A., \& Boz, A. (2007). Exact solutions for a class of nonlinear partial differential equations using exp-function method. International Journal of Nonlinear Sciences and Numerical Simulation, 8(4), 505-512.

[9] Ayhan, B., \& Bekir, A. (2012). The $\left(G^{\prime} / G\right)$-expansion method for the nonlinear lattice equations. Communications in Nonlinear Science and Numerical Simulation, 17(9), 3490-3498.

[10] Naher, H., Abdullah, F. A., \& Bekir, A. (2012). Abundant traveling wave solutions of the compound $\mathrm{KdV}$-Burgers equation via the improved (G?/G)expansion method. AIP Advances, 2(4), 042163.

[11] Wang, D. S., Ren, Y. J., \& Zhang, H. Q. (2005). Further extended sinh-cosh and sin-cos methods and new non traveling wave solutions of the $(2+1)$ dimensional dispersive long wave equations. Appl. Math. E-Notes, 5, 157-163. 
[12] Wazwaz, A. M. (2004). A sine-cosine method for handling nonlinear wave equations. Mathematical and Computer modelling, 40(5-6), 499-508.

[13] Bekir, A. (2008). New solitons and periodic wave solutions for some nonlinear physical models by using the sine-cosine method. Physica Scripta, 77(4), 045008.

[14] Korkmaz, A., \& Hosseini, K. (2017). Exact solutions of a nonlinear conformable time-fractional parabolic equation with exponential nonlinearity using reliable methods. Optical and Quantum Electronics, 49(8), 278.

[15] Hosseini, K., \& Ansari, R. (2017). New exact solutions of nonlinear conformable time-fractional Boussinesq equations using the modified Kudryashov method. Waves in Random and Complex Media, 1-9.

[16] Korkmaz A., On The Wave Solutions of Conformable Fractional Evolution Equations, Commun. Fac. Sci. Univ. Ank. Series A1, 67(1) 68-79, 2018.

[17] Korkmaz A., Exact Solutions to $(3+1)$ Conformable Time Fractional Jimbo-Miwa,Zakharov-Kuznetsov and Modified Zakharov-Kuznetsov Equations, Communications in Theoretical Physics, 2017, 67(5), 479-482.

[18] Kaplan, M., \& Hosseini, K. (2018). Investigation of exact solutions for the Tzitzéica type equations in nonlinear optics. Optik-International Journal for Light and Electron Optics, 154, 393-397.

[19] Hosseini, K., Mayeli, P., \& Ansari, R. (2017). Bright and singular soliton solutions of the conformable time-fractional Klein-Gordon equations with different nonlinearities. Waves in Random and Complex Media, 1-9.

[20] Hosseini, K., Mayeli, P., \& Ansari, R. (2017). Modified Kudryashov method for solving the conformable time-fractional Klein-Gordon equations with quadratic and cubic nonlinearities. Optik-International Journal for Light and Electron Optics, 130, 737-742.

[21] Kaplan, M., Mayeli, P., \& Hosseini, K. (2017). Exact traveling wave solutions of the Wu-Zhang system describing $(1+1)$-dimensional dispersive long wave. Optical and Quantum Electronics, 49(12), 404.

[22] Tasbozan, O., Çenesiz, Y., \& Kurt, A. (2016). New solutions for conformable fractional Boussinesq and combined KdV-mKdV equations using Jacobi elliptic function expansion method. The European Physical Journal Plus, 131(7), 244. 
[23] Çenesiz, Y., Tasbozan, O., \& Kurt, A. (2017). Functional Variable Method for conformable fractional modified KdV-ZK equation and Maccari system. Tbilisi Mathematical Journal, 10(1), 117-125.

[24] Khalil, R., Al Horani, M., Yousef, A., \& Sababheh, M. (2014). A new definition of fractional derivative. Journal of Computational and Applied Mathematics, 264, 65-70.

[25] Morrison, P. J., Meiss, J. D., \& Cary, J. R. (1984). Scattering of regularizedlong-wave solitary waves. Physica D: Nonlinear Phenomena, 11(3), 324-336.

[26] Atangana, A., Baleanu, D., \& Alsaedi, A. (2015). New properties of conformable derivative. Open Mathematics, 13(1), 1-10.

[27] Çenesiz, Y., Baleanu, D., Kurt, A., \& Tasbozan, O. (2016). New exact solutions of Burgers' type equations with conformable derivative. Waves in Random and Complex Media, 1-14.

[28] Abdeljawad, T. (2015). On conformable fractional calculus. Journal of computational and Applied Mathematics, 279, 57-66.

[29] Yan, C. (1996), A simple transformation for nonlinear waves, Physics Letters A, 224, 77-84. 